\title{
Editorial
}

\section{Luxury and counterfeiting: Issues, challenges and prospects}

Journal of Brand Management (2012) 19, 541-543. doi:10.1057/bm.2012.5

Luxury is still alive and well. Indeed, if there is one sector that is currently buoyant, it is, surprisingly, the luxury goods sector. Expecting to generate more than 200 billion euros in worldwide sales in 2012, all luxury industries are experiencing strong annual growth in terms of both value and volume. Over the last two decades, the luxury industry has greatly expanded, with the appearance of new luxury brands, such as certain holiday destinations and airlines. Practitioners and researchers are interested in the increasingly complex behaviour of luxury goods consumers, giving rise to new concepts and research models. The situation is further complicated by fierce competition, heightened by the practice of counterfeiting, which can tarnish the image and profitability of luxury brands. Consequently, understanding consumers' behaviour towards counterfeit products becomes very crucial.

This special issue of the Journal of Brand Management (JBM) includes a selection of papers presented at the first International Conference on Luxury and Counterfeiting organized by Wesford University, Geneva, held in June 2011. The articles in this special issue reflect the diversity and breadth of current research within this stimulating research area. Reflecting the diversity of scholars attending the conference, the authors are from different countries and the papers here vary similarly according to the authors' interests.
The first paper (Luxury consumption in the trade-off between genuine and counterfeit goods: What are the consumers's underlying motives and value-based drivers?) by Wiedmann, Hennigs and Klarmann reviews the reasons why consumers buy genuine luxury brands, what they believe real luxury is, and how their perception of luxury value affects their buying behaviour in the trade-off between authentic or counterfeit products. On the basis of an in-depth literature review, findings reveal that counterfeit luxury products are primarily purchased because of their low price and the widespread opinion that they offer a good value for the money. Hence, it seems that the key challenge is to inform consumers about the risks associated with counterfeit consumption, raise ethical considerations, display the negative consequences for society, and convince them that compared with the value of genuine luxury in the long run counterfeit products are not worth the money.

In the second paper (Does counterfeiting affect luxury customer-based brand equity?), Gabrielli, Grappi and Baghi try to shed some light on the effects of counterfeit awareness on genuine brand users' and on potential users' customer-based brand equity (CBBE). Results are consistent with the adopted theoretical model: Keller's CBBE pyramid. The mean rates of each block are descending from the base to the top of the pyramid, both for genuine brand users' sample and for the non-brand users' 
sample. All in all, counterfeiting awareness does not weaken CBBE, but rather it might strengthen some consumers' perceptions, evaluations and behavioural intentions. In particular, genuine brand consumers show a significant sensibility towards counterfeiting information on almost all of the CBBE dimensions. Knowing that the brand they possess is affected by counterfeit induces them to think that this brand is well performing and to consider it is better than competitors. The incessant efforts made by counterfeiters to try to effectively imitate the style and the quality features of the genuine products (even the warranty and the finishes) probably highlight the uniqueness of the genuine product.

The third paper ( $A$ comparison of the emotional and motivational aspects of luxury products versus counterfeits) by Penz and Stöttinger explores emotional aspects and motivations surrounding the ownership and purchase of fake products in contrast to originals. Findings from eight focus groups in a small European country point to the fact that emotional aspects are important drivers of purchasing decisions for both product categories. Results indicate different patterns of positive, negative or mixed emotions for originals versus fakes. Brands entail positive feelings, for example pride and a sense of accomplishment, and provide the owner/buyer with love from others. Consumers appreciate the product and aesthetic qualities in brands. Counterfeits also turned out to be associated with many positive emotions and motivations. The process of buying fakes seems much more fun and enjoyable; bargaining adds a kick to the consumption experience. Although the shopping process appears less planned and more situation specific, at least some customers prepare it carefully in advance. The only considerable drawback that consumers of fakes take seriously, and which evoked the strongest emotions, is the fear and shame of being detected. The expected social sanctions represent a strong deterrent. The emotions and motivations related to buying counterfeits represent a certain feeling of ease and playfulness; they appeal to a consumer's sense of being a smart shopper and benefit from the positive emotions brands convey.

In the fourth paper, Magnoni and Roux (The impact of step-down line extension on consumer-brand relationships: A risky strategy for luxury brands) explore the step-down line extension effects of core brands from a consumer's perspective. This article examines the role of the brand concept (luxury versus non-luxury) in the impact of step-down line extension on consumer-brand relationships. A before-and-after pseudo-experimental study conducted on the Internet among BMW and Peugeot clients shows that stepdown line extension negatively influences the main variables of consumer-brand relationships (self-brand connections, brand attachment, brand trust and brand commitment) only for the luxury brand BMW. The strongest dilution effects appear for the relational variables of an emotional nature: self-brand connections and brand attachment. On the contrary, no dilution effects are found for the non-luxury brand Peugeot. This research highlights the importance of considering brand concept and consumer-brand relationships in vertical line extensions.

The fifth paper (Why the devil wears Prada: Consumers' purchase motives for luxuries) written by Hudders focuses on both impressive and expressive motives simultaneously and shows that impressive purchase motives (for example, hedonic experiences) for luxury brands are more important than expressive purchase motives (for example, status gains). In addition, results also reveal that both individual differences (that is, materialism level and gender differences) and product characteristics (that is, product conspicuousness) have a moderating impact on the relative importance of these motives. 
This study also reveals that consumers' purchase motives for luxury brands may differ according to the product's level of conspicuousness. Whereas the impressive motives are more important for privately than for publicly consumed luxuries, the expressive purchase motives are equally important for both product categories. These insights into consumers' purchase motives might help luxury marketers to develop effective marketing campaigns.

The last paper (Do consumers perceive three levels of luxury? A comparison of accessible, intermediate and inaccessible luxury brands), by De Barnier, Falcy and Valette-Florence, aims to study the concept of luxury levels and to assess whether they are significant for consumers. Perceptions of five brands were measured: Chanel and Mont Blanc for accessible luxury level; Rolex for intermediary luxury level; and Ferrari and Van Cleef and Arpels for inaccessible luxury level. In addition, three luxury perception scales were compared: Kapferer (1998), Vigneron and Johnson (1999) and Dubois et al (2001). This research shows some convergences between the three scales, as well as the utility of these scales for differentiating luxury brands. Finally, the predictive validity of these scales is unlighted, validating the distinction of brands among the three aforementioned luxury levels. Results also show that there is a luxury continuum at a theoretical level, reinforcing the notions of accessible, intermediate and prototypical inaccessible luxury.

It has been a highly stimulating exercise to edit this special issue on luxury and counterfeiting. The selected articles provide a wide variety of stimulating insights into knowledge advancements in that field. The range of nationalities of the authors, the scope of topics and methodologies used indicate that there is a vibrant and healthy research culture within that field. As a scientific coordinator of this conference and an invited editor of this special issue, I greatly appreciated the inputs of the international scientific committee members of this conference who contributed to the paper selection process and helped guarantee the quality of the contributions. Special thanks also go to Brenda Rouse, the former managing editor of $J B M$, for approving this special issue. Finally, I wish to express my gratitude to Neil Henderson, Senior Publishing Editor at Palgrave Macmillan, now in charge of the Journal of Brand Management, for his diligence and professionalism. Ultimately, special thanks go to Alex Lienard, the President of the Wesford Business Schools Group for initiating this first Conference on Luxury and Counterfeiting.

I hope that you will find the articles in this special issue both enriching and thought-provoking and that the insights provided in the collection of research materials will inspire further interest and research dealing with luxury and counterfeiting.

Pierre Valette-Florence IAE de Grenoble and CERAG, Grenoble, France E-mail: pvalette@upmf-grenoble.fr 